\title{
Treatment-Plan Comparison of Three Advanced Radiation Treatment Modalities for Fractionated Stereotactic Radiotherapy to the Head and Neck
}

\author{
He Wang1, James N. Yang1, Xiaodong Zhang ${ }^{1}$, Jing Li' ${ }^{2}$, Steven J. Frank ${ }^{2}$, Zhongxiang Zhao', \\ Dershan Luo ${ }^{1}$, Xiaorong Zhu', Congjun Wang1, Samuel Tung1, Adam S. Garden², \\ David I. Rosenthal2, Clifton David Fuller2, Gary Brandon Gunn², Amol J. Ghia², Jay P. Reddy', \\ Shaan M. Raza ${ }^{3}$, Franco De Monte ${ }^{3}$, Mark S. Chambers4, Paul D. Brown ${ }^{5}$, Shirley Su4, Jack Phan²* \\ ${ }^{1}$ Department of Radiation Physics, The University of Texas MD Anderson Cancer Center, Houston, TX, USA \\ ${ }^{2}$ Department of Radiation Oncology, The University of Texas MD Anderson Cancer Center, Houston, TX, USA \\ ${ }^{3}$ Department of Neurosurgery, The University of Texas MD Anderson Cancer Center, Houston, TX, USA \\ ${ }^{4}$ Department of Oncologic Dentistry Prosthodontics, Thoracic/Head \&Neck Surgery, The University of Texas MD Anderson \\ Cancer Center, Houston, TX, USA \\ ${ }^{5}$ Department of Radiation Oncology, The Mayo Clinic, Rochester, MN, USA \\ Email: ‘jphan@mdanderson.org
}

How to cite this paper: Wang, H., Yang, J.N., Zhang, X.D., Li, J., Frank, S.J., Zhao, Z.X., Luo, D., Zhu, X.R., Wang, C.J., Tung, S., Garden, A.S., Rosenthal, D.I., Fuller, C.D., Gunn, G.B., Ghia, A.J., Reddy, J.P., Raza, S.M., De Monte, F., Chambers, M.S., Brown, P.D., Su, S. and Phan, J. (2019) Treatment-Plan Comparison of Three Advanced Radiation Treatment Modalities for Fractionated Stereotactic Radiotherapy to the Head and Neck. International Journal of Medical Physics, Clinical Engineering and Radiation Oncology, 8, 106-120. https://doi.org/10.4236/ijmpcero.2019.82010

Received: April 10, 2019

Accepted: May 24, 2019

Published: May 27, 2019

\begin{abstract}
Purpose: Fractionated stereotactic radiosurgery (FSRS) can be given with at least three modalities: Gamma Knife, with the noninvasive frameless extend system (GKE); linear accelerator-based volumetric modulated arc therapy (VMAT); and intensity-modulated proton therapy (IMPT). We extracted treatment plans for patients who had received FSRS with GKE for recurrent skull base or intracranial tumors, created corresponding plans for VMAT and IMPT, and compared the quality of the three sets of plans. Methods and materials: Plans were extracted for 9 patients with recurrent malignant skull-base tumors $(n=6)$ or large intracranial tumors $(n=3)$ who had received FSRS with GKE (median dose 24 Gy in 3 fractions) in 2013 through 2015. Plans for VMAT were generated with a TrueBeam STx LINAC machine using 6-MV photons, and plans for IMPT were generated with multi-field optimization. The optimized VMAT and IMPT plans were normalized to achieve the best possible target coverage while meeting the same dose-volume constraints on organs at risk (OARs) as the GKE plans. Plans were evaluated on the basis of target coverage, conformity index, homogeneity index, gradient index, and treatment efficiency. Results: The median target volume was $10.2 \mathrm{~cm}^{3}$ (range $1.9-33.8 \mathrm{~cm}^{3}$ ). The VMAT and IMPT plans met all OAR con-
\end{abstract}


Copyright $\odot 2019$ by author(s) and Scientific Research Publishing Inc. This work is licensed under the Creative Commons Attribution International License (CC BY 4.0).

http://creativecommons.org/licenses/by/4.0/ straints, and target coverage and conformity were comparable among all plans. VMAT and IMPT plans showed significantly better target uniformity and treatment delivery efficiency $(P<0.001)$. The gradient index and low-dose-bath were superior in the GKE plans $(P<0.001)$, indicating smaller irradiated volumes. When inverse planning was used, VMAT plans could achieve a similar or steeper dose drop-off at the target-OAR boundary than GKE plans. Conclusion: FSRS for skull base and large intracranial lesions delivered by VMAT and IMPT can achieve comparable target coverage, conformity, and sparing of critical structure as the GKE while providing superior target uniformity and treatment delivery efficiency. The GKE had superior high-dose gradients outside the target and thus better protected surrounding normal structures.

\section{Keywords}

Gamma Knife Extend System, Stereotactic Radiosurgery, Volumetric Modulated Arc Therapy, Intensity-Modulated Proton Therapy, Spot Scanning Proton Therapy

\section{Introduction}

Intracranial tumors of a certain size range (i.e. up to about $3 \mathrm{~cm}$ ) are often treated with single-fraction stereotactic radiosurgery (SRS), in which a rapid dose fall-off to spare normal tissues is achieved by using multiple beam directions and a high degree of collimation. The Leksell Gamma Knife Perfexion system (GK), for example, has 192 high-activity Cobalt-60 sources that can be collimated to a single focus, capable of delivering a highly focal and conformal ablative radiation dose with a rapid dose fall-off toward surrounding healthy tissue [1] [2] [3] [4]. With spatial uncertainties of about $0.35 \mathrm{~mm}$, the Perfexion system has an average agreement of $0.1-1.6 \mathrm{~mm}$ for substantial isodose lines [4] and uncertainties in target localization of up to $1.3 \mathrm{~mm}$ for cases involving multiple brain metastases [5]. For maximum accuracy, SRS delivered with this system requires use of a minimally invasive frame that is rigidly mounted onto the patient's skull.

In contrast to single-session SRS, fractionated stereotactic radiosurgery (FSRS), in which radiation is delivered in 3 - 5 large fractions, is an attractive option for larger intracranial tumors or skull base tumors [6] because the single-fraction dose required for tumor control often exceeds the tolerance of adjacent critical neuro-optic structures. Delivery of FSRS with the GK is facilitated by the use of an alternative to the head frame, in which the patient's head position is fixed by means of a customized vacuum-assisted mouthpiece (e.g. the Extend system; Figure 1).

Another means of delivering FSRS is with advanced linear accelerators (LINACs), such as the TrueBeam STx (Varian Medical Systems, Inc., Palo Alto, CA). LINACs have the advantages of sub-millimeter precision, a fully integrated 


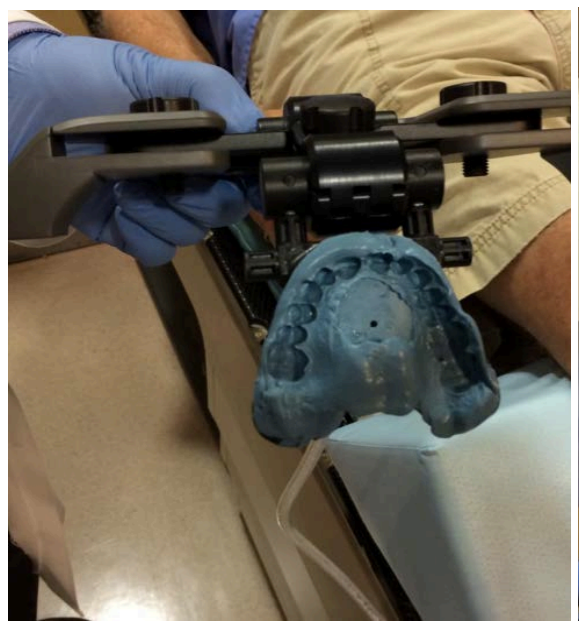

(a)

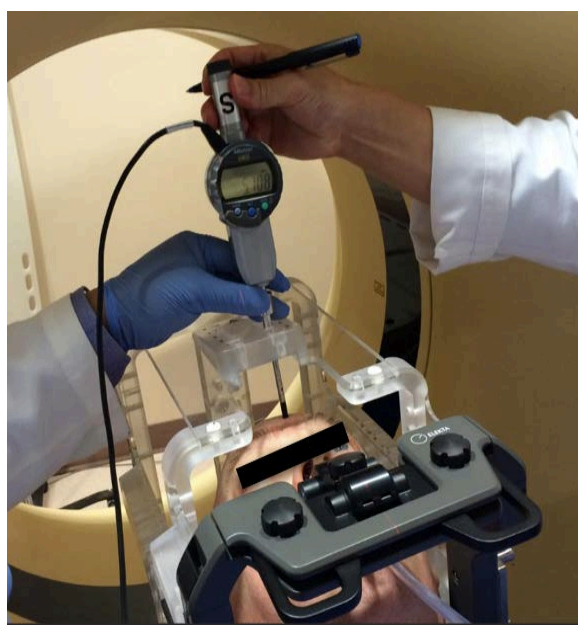

(b)

Figure 1. (a) The Extend System utilizing a vacuum assisted mouthpiece attached to a stereotactic carbon fiber frame for reproducible fixation; (b) Digital reposition check tool.

volumetric imaging system, high-dose-rate delivery, motion management techniques, and high-definition (2.5-mm) multi-leaf collimation [7]. Use of volumetric modulated arc therapy (VMAT) on LINACs also permits continuous delivery of radiation, which can significantly reduce treatment time.

A third means of delivering FSRS is by proton beam therapy. The unique dosimetric characteristic of protons can minimize the dose to normal tissue distal to the tumor relative to that from high-energy photons and can be used for FSRS of head and neck tumors [8] [9]. Recent advances in proton radiation delivery involving spot scanning or intensity-modulated proton therapy (IMPT) [10] [11] [12] [13] enable sufficiently conformal plans to treat head and neck tumors. For this purpose, IMPT has the potential to capture the "best of both worlds" by limiting the high-dose region beyond the tumor while also limiting the low dose bath, i.e. exposure of surrounding tissues to low-dose radiation.

Treatment for a subset of head and neck tumors, i.e. those at the skull base, or larger intracranial tumors is uniquely challenging because of the strict constraints on nearby critical structures. Here, we extracted treatment plans from patients with skull-base or intracranial tumors treated with FSRS, used them to generate plans for VMAT and IMPT, and compared the quality of the three sets of treatment plans.

\section{Methods and Materials}

We first identified 9 patients who had received FSRS with GKE (6 with skull base tumors and 3 with large intracranial tumors) from February 2013 to January 2015 at a single institution (Table 1). Treatment in all cases had been directed to a single isocenter/target, and the median prescription dose was $24 \mathrm{~Gy}$ (range 21 - 27 Gy) delivered in 3 daily fractions.

The GK treatment plans were generated under magnetic resonance imaging (MRI) or computerized tomography (CT) guidance and were obtained within a 
Table 1. Patient and treatment information.

\begin{tabular}{|c|c|c|c|c|c|c|c|c|c|}
\hline \multirow{2}{*}{ Patient } & \multirow{2}{*}{ Specific Site } & \multirow{2}{*}{$\begin{array}{l}\text { Target Volume } \\
\qquad\left(\mathrm{cm}^{3}\right)\end{array}$} & \multirow{2}{*}{$\begin{array}{c}\text { Prescription } \\
\text { (Gy) }\end{array}$} & \multirow{2}{*}{$\begin{array}{l}\text { \# GK } \\
\text { shots }\end{array}$} & \multicolumn{2}{|c|}{ MU } & \multicolumn{3}{|c|}{ Delivery Time (minutes) } \\
\hline & & & & & VMAT & IMPT & GKE & VMAT & IMPT \\
\hline 1 & Right Occiput & 33.8 & 24 & 57 & 3108 & 251 & 74.3 & 5.4 & 7.0 \\
\hline 2 & Right Occiput & 30.1 & 21 & 35 & 1703 & 177 & 43.1 & 3.0 & 6.3 \\
\hline 3 & Left Posterior Lobe & 18.2 & 27 & 33 & 1920 & 166 & 45.9 & 3.4 & 5.1 \\
\hline 4 & Left Sphenoid & 14.1 & 27 & 39 & 2040 & 162 & 65.6 & 3.6 & 7.2 \\
\hline 5 & Right Cerebellum & 10.2 & 24 & 50 & 1619 & 124 & 66.1 & 2.8 & 3.9 \\
\hline 6 & Right Cavernous Sinus & 9.6 & 21 & 36 & 1688 & 115 & 47.2 & 3.0 & 5.1 \\
\hline 7 & Right Petrous Apex & 8.8 & 24 & 33 & 2087 & 95 & 67.1 & 3.7 & 4.2 \\
\hline 8 & Left Frontal Lobe & 2.5 & 24 & 28 & 1687 & 45 & 31.0 & 3.0 & 2.0 \\
\hline 9 & Left Petrous Apex & 1.9 & 21 & 33 & 1938 & 67 & 42.9 & 3.4 & 1.8 \\
\hline
\end{tabular}

Abbreviations: GK, Gamma knife; MU, monitor units; VMAT, volumetric modulated arc therapy; IMPT, intensity-modulated proton therapy.

week before the first treatment session. Plans for GK therapy with the Extend system (GKE) had been generated by a combination of inverse planning and forward planning by a team of radiation oncologists, neurosurgeons, and physicists using the Leksell Gamma Plan 10.1.1 (Elekta, Stockholm, Sweden). After the mouth piece assisted frame was placed, the patient was transported to the simulation CT scanner and a setup verification CT scan was acquired. That scan was then fused to the treatment-planning image to verify the consistency of setup by using the Extend system's repositioning tools (Figure 1(b)). GKE treatment plans included a 2-mm margin around the tumor to form the planning target volume (PTV).

\subsection{VMAT Treatment Planning}

From each GKE plan, a VMAT plan was generated using a Pinnacle treatment planning system (Philips Medical Systems, Fitchburg, WI) on a TrueBeam STx LINAC using 6-MV photons and high-definition multi-leaf collimators. PTVs and organs at risk (OARs) were defined on the GKE plan and digitally transferred to Pinnacle using the DICOM-RT protocol for VMAT planning. The 6-MV beams used in this study had been commissioned for stereotactic radiosurgery dosimetry. Although the VMAT plans were optimized with a 2-mm dose grid to speed up the optimization process, the final dose distributions were calculated in a 1-mm dose grid to ensure comparable accuracy to that of the GK dose calculations [14].

\subsection{IMPT Treatment Planning}

To generate IMPT plans, planning CT scans and contoured structures from the GKE plans were transferred to the Eclipse treatment planning system (Varian Medical Systems, Inc.). The IMPT plans were generated on an Hitachi ProBeat delivery system [15] using 3 - 5 spot-scanning beams, with each spot $>1 \mathrm{~cm}$. In- 
verse planning with multi-field optimization was used as described elsewhere [16] [17].

\subsection{Dosimetric Analysis}

After optimization, VMAT and IMPT plans were normalized to meet same or less dose-volume constraints on organs at risk (brainstem and optical pathway volume at $12 \mathrm{~Gy}$ and $8 \mathrm{~Gy}$ ) as the GKE plans while achieving best possible target coverages. Treatment plans were evaluated with seven metrics: target coverage; Paddick conformity index (CI) [18]; target homogeneity index (HI); gradient index (GI); the irradiated volume-to-PTV ratio $\left(\mathrm{R}_{50}\right)$; the low-dose volume-to-PTV ratio (low-dose volume index, $\mathrm{R}_{20-50}$ ); and treatment efficiency (i.e. the delivery time [dTime] for one fraction). The $\mathrm{HI}$ is defined as the relative standard deviation or coefficient of variation (percentage of one standard deviation to the mean dose) in the PTV. The prescription isodose volume (PIV) is the irradiated volume within the prescription isodose line; Thus PIV $_{50}$ is the irradiated volume enclosed by the $50 \%$ prescription isodose line, and $\mathrm{PIV}_{20-50}$ is the volume receiving between $20 \%$ and $50 \%$ of the prescription isodose. The GI is defined as $\mathrm{PIV}_{50} / \mathrm{PIV}$, representing the extent of dose fall-off at $50 \%$ of the prescription dose. The irradiated volume-to-PTV ratio, $\mathrm{R}_{50}$, is defined as $\mathrm{PIV}_{50} / \mathrm{PTV}$, and the low-dose volume index, $\mathrm{R}_{20-50}$, is defined as the $\mathrm{PIV}_{20-50} / \mathrm{PTV}$. Finally, regarding treatment efficiency, because the time required for patient immobilization and setup is not related to the number of treatment fields/shots, the delivery time is defined as the sum of time for each beam from the start of delivery to completion. The dTime for VMAT was based on a dose rate of 600 monitor units $(\mathrm{MU}) / \mathrm{min}$, that for GKE, on a $3.0 \mathrm{~Gy} / \mathrm{min}$ dose rate, and that for IMPT on the number of layers and spots [19]. Independent sample $t$ tests were used for comparisons, and all statistical analyses were done with IBM SPSS Statistics 22.

\section{Results}

Patient and dosimetric variables are shown in Table 1 . The median target volume for all patients was $10.2 \mathrm{~cm}^{3}$ (range $1.9-33.8 \mathrm{~cm}^{3}$ ). The three intracranial target volumes ranged from $2.5 \mathrm{~cm}^{3}$ to $18.2 \mathrm{~cm}^{3}$, and the six skull-base target volumes ranged from $1.9 \mathrm{~cm}^{3}$ to $33.8 \mathrm{~cm}^{3}$. Both target volumes and OAR volumes increased after being transferred to the Pinnacle (mean 5.1\%, range 0.3\% to $11.2 \%$ ) and the Eclipse (mean 3.9\%, range $0.0 \%$ to $7.8 \%$ ) treatment planning systems (not shown). Because the target volumes were slightly larger in Pinnacle and Eclipse, our goal was to achieve $99.5 \%$ coverage after critical OAR (brainstem and optic pathway) constraints were met.

The Extend positioning error was $\leq 1.0 \mathrm{~mm}$ (3D vector $0.58 \mathrm{~mm} \pm 0.35 \mathrm{~mm}$ ) based on pretreatment CT images. The mean number of shots for the GKE was 38.2 (range 28 - 57), and the mean numbers of MUs were 1976.7 (range 1619 3108 MUs) for the VMAT and 33.6 (range 45 - 251 MUs) for the IMPT (Table 1). No correlation was found between shot number and MUs. Representative GKE, VMAT and IMPT plans are shown in Figure 2, and dose-volume histo- 
gram analysis of the patient depicted in Figure 2(a) is shown in Figure 3.

\section{Dosimetric Comparison}

Comparisons of the dosimetric characteristics of each of the three sets of plans are shown numerically in Table 2 and graphically in Figure 4. Overall, the mean target coverage across all patients was similar for the GKE, VMAT, and IMPT plans, with $98.9 \% \pm 2.4 \%$ coverage (range $93.0 \%$ to $100 \%$ ) for GKE, $99.4 \% \pm$ $0.6 \%$ coverage (range $98.1 \%$ to $100.0 \%$ ) for VMAT, and $96.8 \% \pm 2.1 \%$ coverage (range $93.6 \%$ to $99.5 \%$ ) for IMPT. However, for targets smaller than $15 \mathrm{~cm}^{3}$, we could not achieve the goal of $99.5 \%$ coverage while also meeting the constraints on OARs for IMPT plans (mean $96.8 \%$, range $94.1 \%$ to $98.5 \%$ ). For VMAT plans, achieving $>99.5 \%$ target coverage was not problematic for targets of $<15 \mathrm{~cm}^{3}$ (mean $99.9 \%$, range $99.5 \%$ to $100 \%)(P<0.05$ vs IMPT).

The conformity indices (CIs) were comparable among all plans $(0.67 \pm 0.11$ for GKE, $0.68 \pm 0.08$ for VMAT, and $0.71 \pm 0.12$ for IMPT). The CI did not vary significantly by target volume or location (intracranial vs. skull base). As indicated by the gradient index (GI), GKE plans $(2.8 \pm 0.2 ; P<0.001)$ had a sharper dose fall-off than did VMAT $(3.9 \pm 0.7)$ or IMPT $(5.2 \pm 1.7)$. GKE plans also had the least low-dose spillage, with an $\mathrm{R}_{50}$ of $4.1 \pm 0.8$ versus $5.7 \pm 1.1$ for VMAT and $7.5 \pm 4.5$ for IMPT $(7.5 \pm 4.5)(P<0.001)$, and with an $\mathrm{R}_{20-50}$ of $12.0 \pm 1.9$ versus $20.2 \pm 8.3$ for VMAT and $20.6 \pm 19.6$ for IMPT $(P<0.001)$. Among the VMAT and IMPT plans, gradient index, and $\mathrm{R}_{20-50}$ seemed to increase with decreasing target volume (Figure 4). However, this trend was not observed for GKE plans.

VMAT plans were among the most homogeneous plans, with HI values within the target of $4.6 \% \pm 1.7 \%$ for VMAT, $4.8 \% \pm 1.7 \%$ for IMPT, and $13.7 \% \pm$ $1.5 \%$ for GKE plans. The normalization value to maximum dose was $78.2 \% \pm$ $4.2 \%$ for VMAT, $80.9 \pm 4.1$ for IMPT, and $49.0 \% \pm 2.0 \%$ for GKE plans. Delivery time (dTime) was significantly shorter for VMAT $(3.5 \pm 0.8 \mathrm{~min})$ and for IMPT $(4.7 \pm 2.0 \mathrm{~min})$ than for GKE $(53.7 \pm 14.8 \mathrm{~min}, P<0.001)$.

\section{Discussion}

In this study, we generated comparison VMAT and IMPT plans for patients with skull-base or large intracranial lesions that had been treated with GKE to a median dose of $24 \mathrm{~Gy}$ in 3 fractions. Our goals were to meet the critical OAR dose constraints of the GKE plans and to quantitatively evaluate VMAT and IMPT plan quality for SRT. To the best of our knowledge, no such findings have been published comparing GK, VMAT and IMPT for FSRS of the head and neck. We found that VMAT and IMPT plans could achieve similar or lower doses to critical OARs compared with the GKE plans, with comparable target coverage. VMAT and IMPT offered more dose-homogeneous plans and significantly shorter delivery time (about 4 minutes for VMAT or IMPT vs. about 53 minutes for GKE). By contrast, the GKE plans had a higher GI and less low-dose spillage than did VMAT or IMPT, indicating less irradiation of normal tissues. 

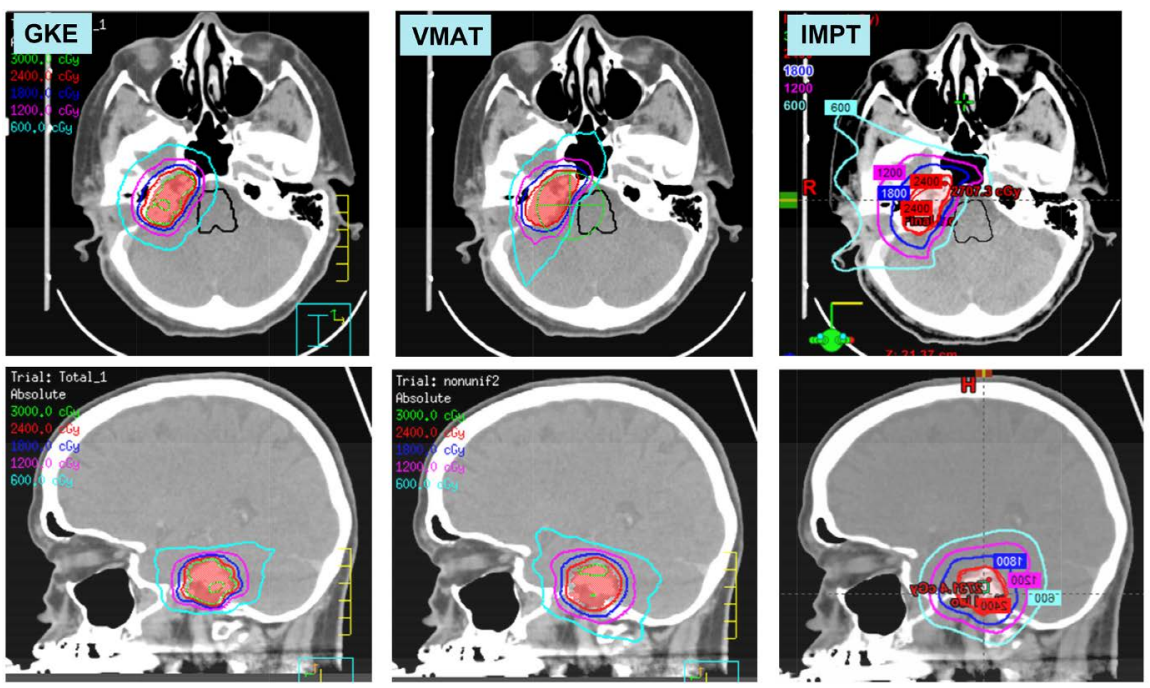

(a)
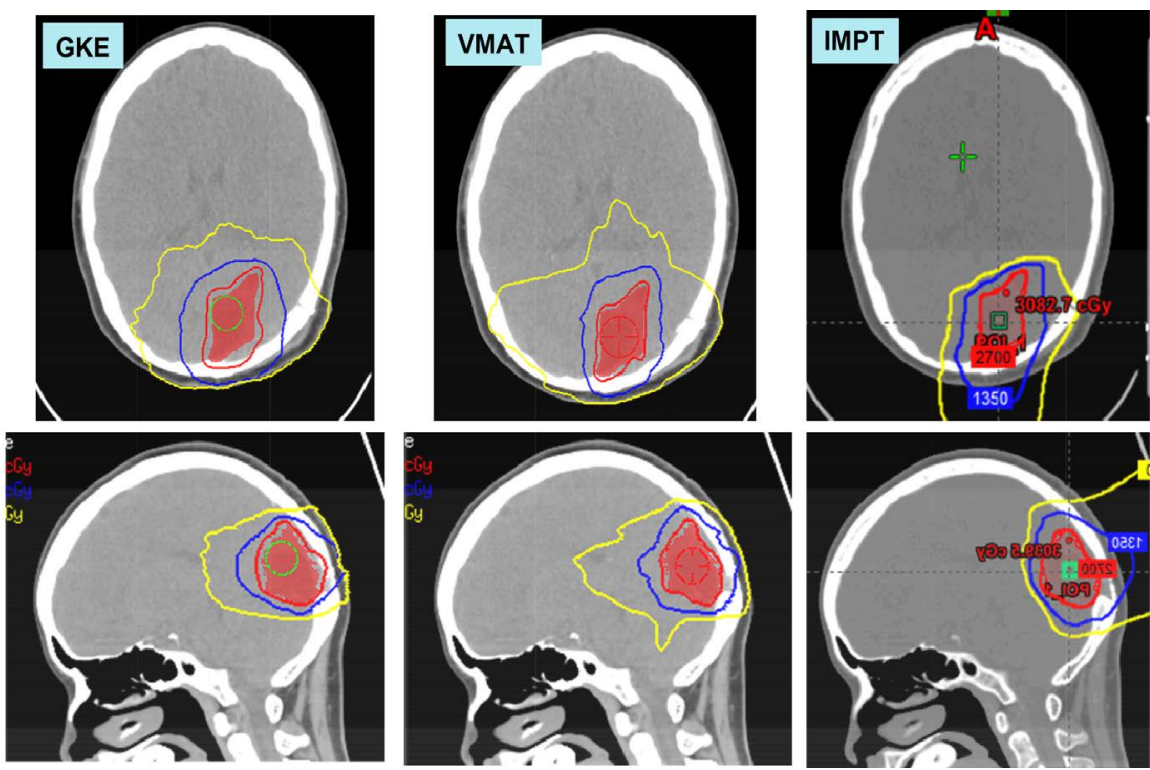

(b)

Figure 2. Dose distribution of a skull-base patient (Panel a) and a intracranial target patient (Panel b). For each panel, from left to right: dose distribution of GKE, VMAT and IMPT plans in axial (top row) and sagittal (bottom row) planes. (a) Right skull base lesion in Patient \#7. Dose lines are 25 Gy (red; prescription dose), 18 Gy (blue), 12 Gy (purple), and $6 \mathrm{~Gy}$ (cyan). On axial image of VMAT plan, the green crosshair on axial VMAT images show isocenter placement at the boundary of lesion and brainstem to achieve a sharp dose gradient. (b) Left posterior lobe surgical bed in Patient \#3. Dose lines are 27 Gy (red), 13.5 Gy (blue), and 5.4 Gy (yellow). For superficially located targets, IMPT plans showed excellent sparing of normal tissues with a sharp gradient and minimal low dose bath.

This difference in favor of GKE compared with VMAT and IMPT was the most pronounced for the treatment of smaller tumors.

In instances where the dose for single-session radiosurgery would exceed the tolerance of a nearby critical structure, FSRS is preferred. Before the wide 


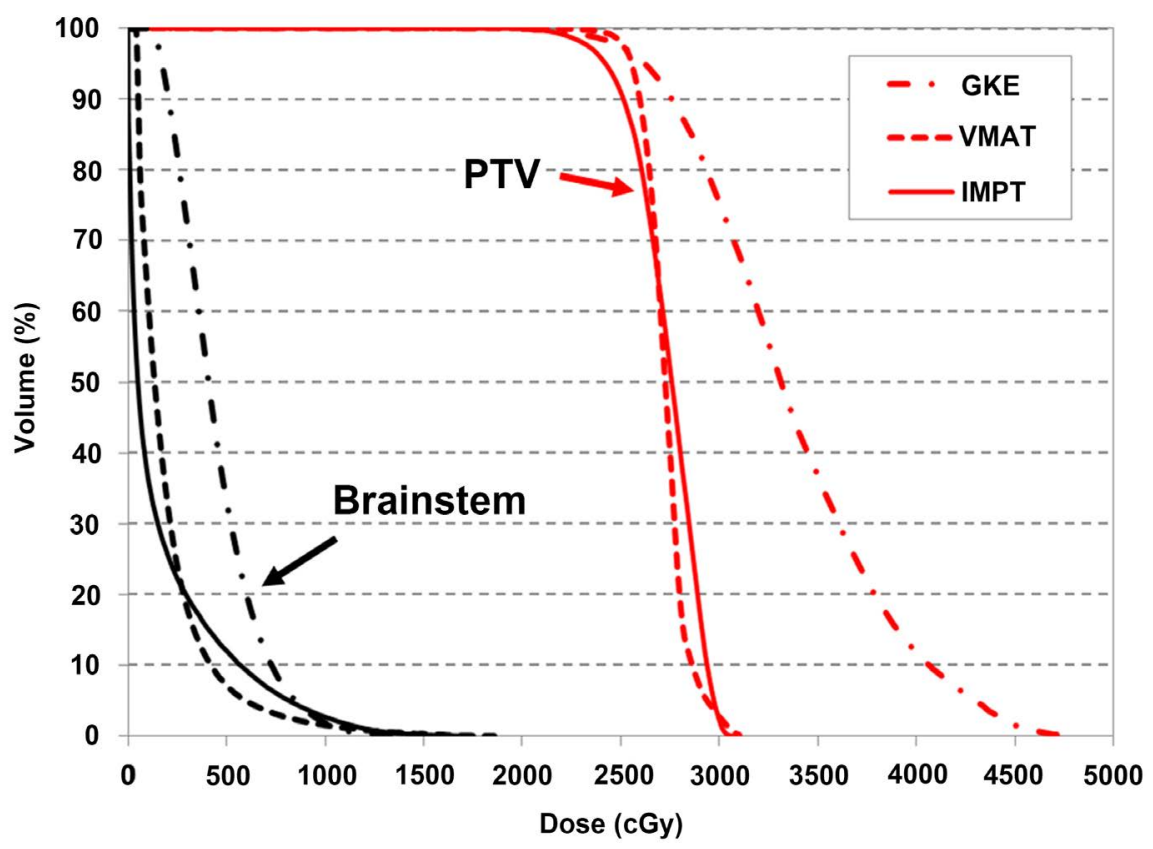

Figure 3. Dose volume histogram of target and brainstem for GKE, VMAT and IMPT plans for patient \#7.

Table 2. Dosimetric comparison of VMAT and IMPT plans to GKE plans.

\begin{tabular}{|c|c|c|c|c|}
\hline & GKE & $\begin{array}{c}\text { VMAT } \\
(P \text { Value })\end{array}$ & $\begin{array}{c}\text { IMPT } \\
(P \text { Value })\end{array}$ & $\begin{array}{c}P \text { value } \\
\text { VMAT vs. IMPT }\end{array}$ \\
\hline PTV coverage $(\%)$ & $98.9 \pm 2.4$ & $\begin{array}{c}99.4 \pm 0.6 \\
(0.451)\end{array}$ & $\begin{array}{c}96.8 \pm 2.1 \\
(0.060)\end{array}$ & 0.005 \\
\hline $\mathrm{CI}$ & $0.67 \pm 0.11$ & $\begin{array}{c}0.68 \pm 0.08 \\
(0.122)\end{array}$ & $\begin{array}{c}0.71 \pm 0.12 \\
(0.440)\end{array}$ & 0.519 \\
\hline GI & $2.8 \pm 0.2$ & $\begin{array}{c}3.9 \pm 0.7^{*} \\
(<0.001)\end{array}$ & $\begin{array}{c}5.2 \pm 1.7^{*} \\
(0.003)\end{array}$ & 0.003 \\
\hline HI (\%) & $13.7 \pm 1.5$ & $\begin{array}{l}4.6 \pm 1.7^{*} \\
(<0.001)\end{array}$ & $\begin{array}{c}4.8 \pm 1.7^{*} \\
(<0.001)\end{array}$ & 0.265 \\
\hline Delivery time (min) & $53.7 \pm 14.8$ & $\begin{array}{c}3.5 \pm 0.8^{*} \\
(<0.001)\end{array}$ & $\begin{array}{l}4.7 \pm 2.0^{*} \\
(<0.001)\end{array}$ & 0.059 \\
\hline Normalization (\%) & $49.0 \pm 2.0$ & $\begin{array}{c}78.2 \pm 4.2^{*} \\
(<0.001)\end{array}$ & $\begin{array}{c}80.9 \pm 4.1^{*} \\
(<0.001)\end{array}$ & 0.606 \\
\hline Irradiated volume/PTV & $4.1 \pm 0.8$ & $\begin{array}{c}5.7 \pm 1.1^{*} \\
(0.008)\end{array}$ & $\begin{array}{c}7.5 \pm 4.5^{*} \\
(0.031)\end{array}$ & 0.219 \\
\hline Low dose volume/PTV & $12.0 \pm 1.9$ & $\begin{array}{c}20.2 \pm 8.3^{*} \\
(0.022)\end{array}$ & $\begin{array}{c}20.6 \pm 19.6 \\
(0.189)\end{array}$ & 0.905 \\
\hline
\end{tabular}

*, $P$ value $<0.05$ compared to GKE. Abbreviations: GKE, Gamma Knife Extend; VMAT, volumetric modulated arc therapy; IMPT, intensity-modulated proton therapy; PTV, planning target volume

availability of LINAC-based SRT systems, the Extend system was used on a GK unit to avoid the need for repeated head frame placement. The measurement system consists of a repositioning digital check tool attached to the Extend frame (Figure 1), which can position a patient to within $1.0 \mathrm{~mm}$ of the reference position [20] [21]. With a calculated mean interfractional radial setup difference of 

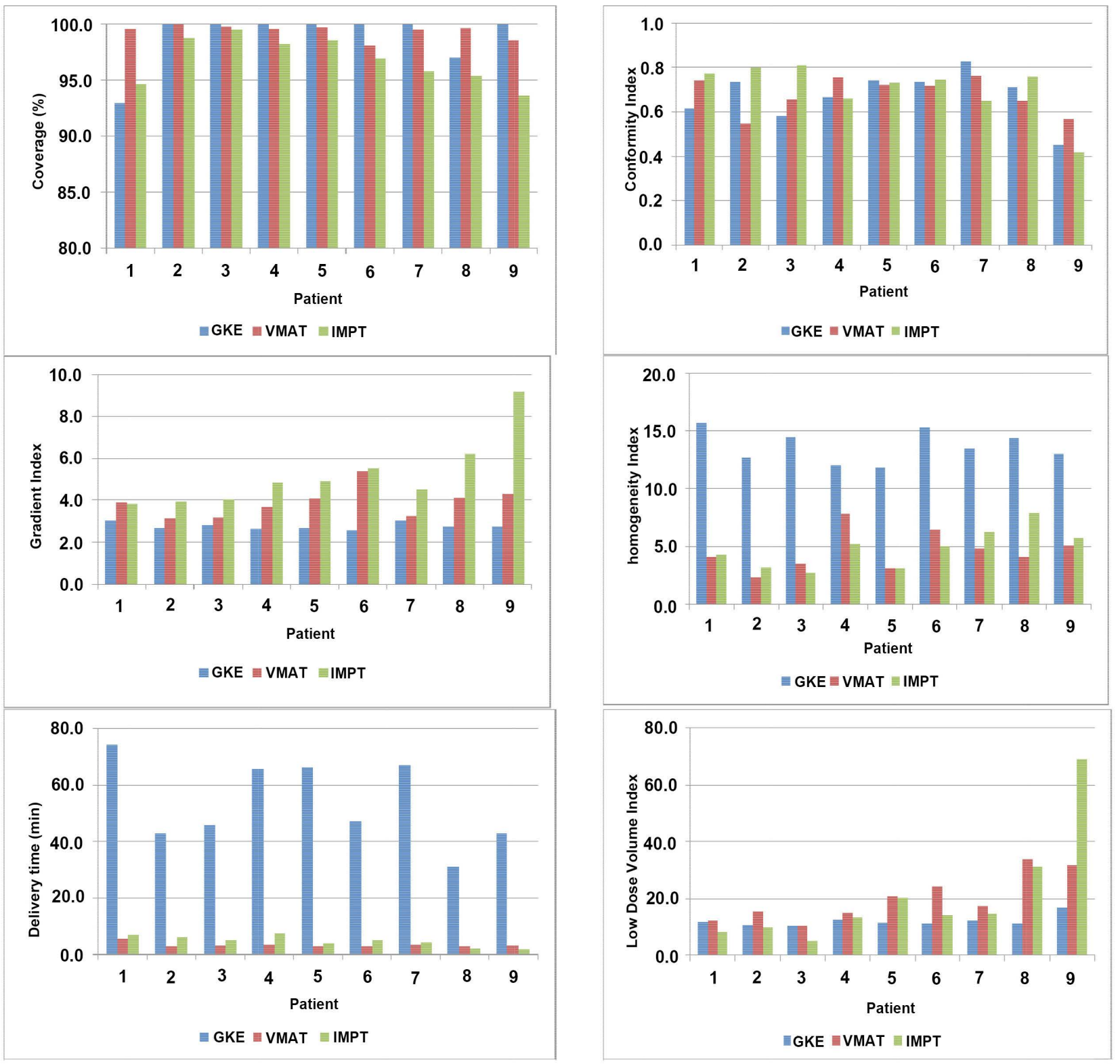

Figure 4. Comparison of dosimetry and treatment efficiency for GKE, VAMT and IMPT plans. PTV volumes see Table 1. Patients are ordered from largest volume (Patient \#1: $33.8 \mathrm{~cm}^{3}$ ) to smallest (Patient \#9: $1.9 \mathrm{~cm}^{3}$ ).

$0.64 \pm 0.24 \mathrm{~mm}$ and an intrafractional positional difference of $0.47 \pm 0.3 \mathrm{~mm}$, the overall setup and immobilization uncertainty of GKE is between 1.0 and $2.0 \mathrm{~mm}$ [21]. Use of the Extend system on a LINAC with only a reposition check tool confers a mean 3D positional displacement of $1.3 \mathrm{~mm}$ for patient setup [22], and so a 2 -mm uncertainty margin for patients treated with the GKE system is typically used to ensure adequate coverage. Today, LINACs such as the TrueBeam STx are used for frameless FSRS and can achieve sub-millimeter imaging setup accuracy, comparable to that of the GKE system. Advantages of LINAC systems are their integrated volumetric imaging system, easy verification of daily patient treatment positioning before and during treatment, and their ability to detect 
and correct setup errors before and during treatment. Thus for VMAT, use of a 2-mm PTV should ensure adequate target coverage while still accommodating machine uncertainties. Indeed, several studies have shown that a practical margin of $<1.5 \mathrm{~mm}$ on the TrueBeam STx is sufficient because of the accuracy of measured isocenter and multi-leaf collimator positioning, a coincidence of $\mathrm{kV}$ and MV imaging isocenters that is within $1.0 \mathrm{~mm}$ [23], and geometric margins that are within $\sim 1.2 \mathrm{~mm}$ [24]. Two dosimetric studies have compared GK with LINAC-based VMAT for single-session treatment of intracranial lesions; in these studies, McDonald et al. [25] and Thomas et al. [26] found comparable target coverage and conformity for treating patients with multiple brain metastases. Our results for FSRS for skull base tumors demonstrate that VMAT can achieve the same or even steeper dose drop-off towards priority critical organs such as the brainstem and optic apparatus compared with the GKE (see Figure 2 ). This was typically achieved by placing the isocenter between the target volume and the critical OAR (Figure 2(a), axial image for VMAT plan) and subsequently using a half-beam block technique with a non-coplanar arc.

Proton beam therapy may also be well suited for skull base FSRS. Published dosimetric comparisons of IMRT/VMAT and IMPT for patients with head and neck tumors treated with conventional fractionation have suggested that IMPT has better conformity [10] [13] [27] [28]. To our knowledge, studies evaluating proton-based FSRS for skull-base or head and neck tumors have not been reported. Here, we used a 2-mm PTV for VMAT and a 2-mm "optimization target volume" for IMPT. Although our results indicate that proton beam therapy for skull base FSRS is feasible, uncertainties in addition to patient setup and machine mechanical limitations should be considered. Of particular importance are particle beam range uncertainties, which are influenced by factors such as CT artifacts, conversion of Hounsfield units to stopping powers, or changes in patient geometry [29] [30] [31]. Thus, a limitation not addressed in this study is the potential requirement for a relatively larger "PTV" margin for proton SRT [32] [33]. An uncertainty of $3.5 \%+1 \mathrm{~mm}$ is often considered during proton treatment planning for conventionally fractionated treatments [30] [34] [35]. We observed that IMPT plans showed excellent sparing of normal tissues, with a sharp gradient index and minimal low-dose bath for larger and superficially located targets (patients 1, 2 and 3 [patient 3 shown in Figure 2]). Improved CT image quality, accurate patient setup verification, and high-quality treatment plans that are less sensitivity to the aforementioned uncertainties continue to be sought for proton radiotherapy to minimize overshoot [30] [34] [35].

A clear advantage of VMAT and IMPT over GKE is the significantly shorter delivery time. The typical treatment time for each fraction with VMAT on a TrueBeam machine was less than 10 minutes, including the delivery time from the first beam to end of treatment, gantry and couch setup, and imaging verification between beams. The delivery time for IMPT was mainly based on total number of the spot and layers. The overall treatment time (including beam re- 
quest time and wait time between beams) may be up to 20 min when 3 - 4 beams are used [19]. A marked difference between the GKE and the VMAT or IMPT plans was observed in dose heterogeneity, in that the HI of GKE plans (13.7\%) was significantly greater than that for VMAT $(4.6 \% ; P<0.05)$ or IMPT $(4.8 \% ; P$ $<0.05)$. The difference in the HI between GKE and VMAT/IMPT was not influenced by tumor size or location.

GKE plans showed a superior GI and better low-dose-volume index $\left(\mathrm{R}_{20-25}\right)$, indicating a sharper dose falloff outside the target. In fact, the $\mathrm{R}_{20-50}$ for GKE was only about half that of VMAT and IMPT plans, because the GK unit produces less lateral scattering of lower energy radiation (Co-60) and has shorter distances from source and collimator to focus. Despite these differences in machine capabilities, we found that with inverse planning, VMAT could achieve similar or lower doses to OAR without loss of coverage; moreover the dose gradient at the OAR-target boundary can be further optimized for steeper drop-off if necessary. By contrast, IMPT achieved slightly lower coverage with similar OAR constraints, and the dose gradient was often not as favorable as that of VMAT, perhaps because the spot size of the proton system at our institution is larger than 1 $\mathrm{cm}$; this could also explain the marked increase in the low-dose bath and higher GI for small and centrally located lesions for IMPT plans (patients 6 - 9). Currently, stereotactic application of protons may be more appropriate for superficial targets if the same radiation treatment and plan quality can be maintained. Limitations of the current study include selection bias owing to the small number of patients selected specifically for GKE treatment. The Extend involves use of a vacuum-sealed, custom prosthesis that locks onto the teeth and can be challenging to tolerate. At our institution, use of this system requires that the patient have good performance status, at least 3 intact teeth, and no gag reflex. We did not use the Extend frame for LINAC- or IMPT-based FSRS. If we had, the repositioning check tool would have been used before each treatment to correct interfractional setup error. Thus use of our current thermoplastic head and neck immobilization system with LINAC- or IMPT-based FSRS may have involved setup differences [36]. Further comparisons of the two systems are needed with regard to intrafractional translational and rotational restriction of patients, particularly for skull base targets, which are often adjacent to the neuro-optic apparatus. In addition, it is unclear if the differences in low-dose bath we observed among VMAT, IMPT, and GKE are clinically significant for FSRS. A previous study of 130 patients with head and neck cancer treated with conventionally fractionated IMRT indicated that radiation-induced nausea and vomiting were significantly associated with mean doses to particular nausea-associated regions [37]. Clinical studies are needed to determine the clinical impact, if any, of the low-dose bath and differences in the other dosimetric variables found in this study for FSRS for head and neck tumors.

\section{Conclusion}

LINAC-based VMAT plans and spot-scanning IMPT plans can achieve compa- 
rable target coverage and conformity as GKE plans for FSRS, with better target uniformity and superior treatment delivery efficiency. GKE plans had the highest dose gradient outside the target and hence provide better protection of normal tissues outside the prescription volume.

\section{Conflicts of Interest}

The authors declare no conflicts of interest regarding the publication of this paper.

\section{References}

[1] Mack, A., Czempiel, H., Kreiner, H.-J., et al. (2002) Quality Assurance in Stereotactic Space. A System Test for Verifying the Accuracy of Aim in Radiosurgery. Medical Physics, 29, 561-568. https://doi.org/10.1118/1.1463062

[2] Novotny, J., Bhatnagar, J.P., Xu, Y., et al. (2014) Long-Term Stability of the Leksell

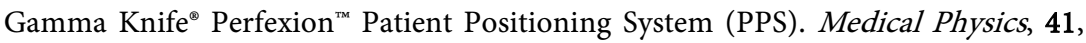
Article ID: 031711. https://doi.org/10.1118/1.4866225

[3] Ma, L., Chiu, J., Hoye, J., et al. (2014) Quality Assurance of Stereotactic Alignment and Patient Positioning Mechanical Accuracy for Robotized Gamma Knife Radiosurgery. Physics in Medicine and Biology, 59, N221.

https://doi.org/10.1088/0031-9155/59/23/N221

[4] Ma, L., Chuang, C., Descovich, M., et al. (2008) Whole-Procedure Clinical Accuracy of Gamma Knife Treatments of Large Lesions. Medical Physics, 35, 5110-5114. https://doi.org/10.1118/1.2987669

[5] Karaiskos, P., Moutsatsos, A., Pappas, E., et al. (2014) A Simple and Efficient Methodology to Improve Geometric Accuracy in Gamma Knife Radiation Surgery: Implementation in Multiple Brain Metastases. International Journal of Radiation Oncology, Biology, Physics, 90, 1234-1241.

https://doi.org/10.1016/j.ijrobp.2014.08.349

[6] Kress, M.-A.S., Sen, N., Unger, K.R., et al. (2015) Safety and Efficacy of Hypofractionated Stereotactic Body Reirradiation in Head and Neck Cancer: Long-Term Follow-Up of a Large Series. Head \& Neck, 37, 1403-1409.

https://doi.org/10.1002/hed.23763

[7] Tanyi, J.A., Summers, P.A., McCracken, C.L., et al. (2009) Implications of a High-Definition Multileaf Collimator (HD-MLC) on Treatment Planning Techniques for Stereotactic Body Radiation Therapy (SBRT): A Planning Study. Radiation Oncology, 4, 22. https://doi.org/10.1186/1748-717X-4-22

[8] Smith, A.R. (2009) Vision 20/20: Proton Therapy. Medical Physics, 36, 556-568. https://doi.org/10.1118/1.3058485

[9] Chhabra, A., Langen, K. and Mehta, M.P. (2016) An Overview of Modern Proton Therapy. Chinese Clinical Oncology, 5, 48. https://doi.org/10.21037/cco.2016.05.06

[10] Apinorasethkul, O., Kirk, M., Teo, K., et al. (2017) Pencil Beam Scanning Proton Therapy vs. Rotational Arc Radiation Therapy: A Treatment Planning Comparison for Postoperative Oropharyngeal Cancer. Medical Dosimetry, 42, 7-11. https://doi.org/10.1016/j.meddos.2016.09.004

[11] Steneker, M., Lomax, A. and Schneider, U. (2006) Intensity Modulated Photon and Proton Therapy for the Treatment of Head and Neck Tumors. Radiotherapy and Oncology, 80, 263-267. https://doi.org/10.1016/j.radonc.2006.07.025 
[12] van de Water, T.A., Lomax, A.J., Bijl, H.P., et al. (2012) Using a Reduced Spot Size for Intensity-Modulated Proton Therapy Potentially Improves Salivary Gland-Sparing in Oropharyngeal Cancer. International Journal of Radiation Oncology, Biology, Physics, 82, e313-e319. https://doi.org/10.1016/j.ijrobp.2011.05.005

[13] Welsh, J., Gomez, D., Palmer, M.B., et al. (2011) Intensity-Modulated Proton Therapy Further Reduces Normal Tissue Exposure during Definitive Therapy for Locally Advanced Distal Esophageal Tumors: A Dosimetric Study. International Journal of Radiation Oncology, Biology, Physics, 81, 1336-1342. https://doi.org/10.1016/j.ijrobp.2010.07.2001

[14] Liu, M., Snyder, K., Zhao, B., et al. (2015) In VMAT of Spine Stereotactic Radiosurgery a $1 \mathrm{Mm}$ Grid Size Increases Dose Gradient and Lowers Cord Dose Significantly Relative to a 2.5 Mm Grid Size. Medical Physics, 42, 3446.

https://doi.org/10.1118/1.4924849

[15] Gillin, M.T., Sahoo, N., Bues, M., et al. (2010) Commissioning of the Discrete Spot Scanning Proton Beam Delivery System at the University of Texas M.D. Anderson Cancer Center, Proton Therapy Center, Houston. Medical Physics, 37, 154-163. https://doi.org/10.1118/1.3259742

[16] Holliday, E.B. and Frank, S.J. (2016) Proton Therapy for Nasopharyngeal Carcinoma. Chinese Clinical Oncology, 5, 25. https://doi.org/10.21037/cco.2016.03.05

[17] Kohno, R., Cao, W., Yepes, P., et al. (2017) Dose Comparison between Eclipse Dose Calculation and Fast Dose Calculator in Single- and Multi-Field Optimization Intensity-Modulated Proton Therapy Plans with Various Multi-Beams for Brain Cancer. International Journal of Medical Physics, Clinical Engineering and Radiation Oncology, 6, 12.

[18] Paddick, I. (2000) A Simple Scoring Ratio to Index the Conformity of Radiosurgical Treatment Plans. Technical Note. Journal of Neurosurgery, 93, 219-222. https://doi.org/10.3171/jns.2000.93.supplement_3.0219

[19] Suzuki, K., Palmer, M.B., Sahoo, N., et al. (2016) Quantitative Analysis of Treatment Process Time and Throughput Capacity for Spot Scanning Proton Therapy. Medical Physics, 43, 3975-3986. https://doi.org/10.1118/1.4952731

[20] Sayer, F.T., Sherman, J.H., Yen, C.P., et al. (2011) Initial Experience with the eXtend System: A Relocatable Frame System for Multiple-Session Gamma Knife Radiosurgery. World Neurosurgery, 75, 665-672. https://doi.org/10.1016/j.wneu.2010.12.051

[21] Schlesinger, D., Xu, Z., Taylor, F., et al. (2012) Interfraction and Intrafraction Performance of the Gamma Knife Extend System for Patient Positioning and Immobilization. Special Supplements, 117, 217-224. https://doi.org/10.3171/2012.6.GKS12989

[22] Ruschin, M., Nayebi, N., Carlsson, P., et al. (2010) Performance of a Novel Repositioning Head Frame for Gamma Knife Perfexion and Image-Guided Linac-Based Intracranial Stereotactic Radiotherapy. International Journal of Radiation Oncology, Biology, Physics, 78, 306-313. https://doi.org/10.1016/j.ijrobp.2009.11.001

[23] Clivio, A., Vanetti, E., Rose, S., et al. (2015) Evaluation of the Machine Performance Check Application for TrueBeam Linac. Radiation Oncology, 10, 97. https://doi.org/10.1186/s13014-015-0381-0

[24] Murphy, M.J. (2009) Intrafraction Geometric Uncertainties in Frameless Image-Guided Radiosurgery. International Journal of Radiation Oncology, Biology, 
Physics, 73, 1364-1368. https://doi.org/10.1016/j.ijrobp.2008.06.1921

[25] McDonald, D., Schuler, J., Takacs, I., et al. (2014) Comparison of Radiation Dose Spillage from the Gamma Knife Perfexion with That from Volumetric Modulated Arc Radiosurgery during Treatment of Multiple Brain Metastases in a Single Fraction. Journal of Neurosurgery, 121, 51-59. https://doi.org/10.3171/2014.7.GKS141358

[26] Thomas, E.M., Popple, R.A., Wu, X., et al. (2014) Comparison of Plan Quality and Delivery Time between Volumetric Arc Therapy (RapidArc) and Gamma Knife Radiosurgery for Multiple Cranial Metastases. Neurosurgery, 75, 409-417. https://doi.org/10.1227/NEU.0000000000000448

[27] Kandula, S., Zhu, X., Garden, A.S., et al. (2013) Spot-Scanning Beam Proton Therapy vs Intensity-Modulated Radiation Therapy for Ipsilateral Head and Neck Malignancies: A Treatment Planning Comparison. Medical Dosimetry, 38, 390-394. https://doi.org/10.1016/j.meddos.2013.05.001

[28] van de Water, T.A., Lomax, A.J., Bijl, H.P., et al. (2011) Potential Benefits of Scanned Intensity-Modulated Proton Therapy versus Advanced Photon Therapy with Regard to Sparing of the Salivary Glands in Oropharyngeal Cancer. International Journal of Radiation Oncology, Biology, Physics, 79, 1216-1224. https://doi.org/10.1016/j.ijrobp.2010.05.012

[29] Bokrantz, R. and Fredriksson, A. (2017) Scenario-Based Radiation Therapy Margins for Patient Setup, Organ Motion, and Particle Range Uncertainty. Physics in Medicine and Biology, 62, 1342-1357. https://doi.org/10.1088/1361-6560/aa524d

[30] Paganetti, H. (2012) Range Uncertainties in Proton Therapy and the Role of Monte Carlo Simulations. Physics in Medicine and Biology, 57, R99-R117. https://doi.org/10.1088/0031-9155/57/11/R99

[31] Warren, S., Partridge, M., Bolsi, A., et al. (2016) An Analysis of Plan Robustness for Esophageal Tumors: Comparing Volumetric Modulated Arc Therapy Plans and Spot Scanning Proton Planning. International Journal of Radiation Oncology, Biology, Physics, 95, 199-207. https://doi.org/10.1016/j.ijrobp.2016.01.044

[32] Grant, J.D. and Chang, J.Y. (2014) Proton-Based Stereotactic Ablative Radiotherapy in Early-Stage Non-Small-Cell Lung Cancer. Biomedical Research International, 2014, Article ID: 389048. https://doi.org/10.1155/2014/389048

[33] Register, S.P., Zhang, X., Mohan ,R., et al. (2011) Proton Stereotactic Body Radiation Therapy for Clinically Challenging Cases of Centrally and Superiorly Located Stage I Non-Small-Cell Lung Cancer. International Journal of Radiation Oncology, Biology, Physics, 80, 1015-1022. https://doi.org/10.1016/j.ijrobp.2010.03.012

[34] Schuemann, J., Dowdell, S., Grassberger, C., et al. (2014) Site-Specific Range Uncertainties Caused by Dose Calculation Algorithms for Proton Therapy. Physics in Medicine and Biology, 59, 4007-4031.

https://doi.org/10.1088/0031-9155/59/15/4007

[35] Unkelbach, J., Bortfeld, T., Martin, B.C., et al. (2009) Reducing the Sensitivity of IMPT Treatment Plans to Setup Errors and Range Uncertainties via Probabilistic Treatment Planning. Medical Physics, 36, 149-163.

https://doi.org/10.1118/1.3021139

[36] Wang, H., Wang, C., Tung, S., et al. (2016) Improved Setup and Positioning Accuracy Using a Three-Point Customized Cushion/Mask/Bite-Block Immobilization System for Stereotactic Reirradiation of Head and Neck Cancer. Journal of Applied Clinical Medical Physics, 17, 6038. https://doi.org/10.1120/jacmp.v17i3.6038

[37] Kocak-Uzel, E., Gunn, G.B., Colen, R.R., et al. (2014) Beam Path Toxicity in Candi- 
date Organs-at-Risk: Assessment of Radiation Emetogenesis for Patients Receiving Head and Neck Intensity Modulated Radiotherapy. Radiotherapy and Oncology, 111, 281-288. https://doi.org/10.1016/j.radonc.2014.02.019 